\title{
Synthesis and Aromatase-Inhibitory Activity of Imidazolyl-1,3,5-triazine Derivatives
}

\author{
Toshiyuki Matsuno, ${ }^{*}$ Masanobu Kato, Yoshio Tsuchida, Masayuki Takahashi, \\ Sin-ichi YaGUCHI, and Sumio TERADA
}

Research Laboratory, Zenyaku Kogyo Co., Ltd., 2-33-7, Ohizumi-machi, Nerima-ku, Tokyo 178, Japan.

Received May 27, 1996; accepted October 8, 1996

\begin{abstract}
Triamino-substituted 1,3,5-triazine derivatives were synthesized and tested for inhibitory activities against the aromatase of human placental microsomes and the cytochrome $\mathbf{P 4 5 0}$ side chain cleavage of cholesterol $\left(\mathbf{P 4 5 0}_{\mathrm{SCC}}\right)$ of pig adrenal mitochondria. The compounds having imidazolyl and tertiary amino groups as substituents in the 1,3,5-triazine ring showed significant aromatase-inhibitory activity. Among them, compounds 17, 23, 26, 27 and 28 were more active than the reference compound, CGS 16949A. The inhibitory activities of these compounds against $\mathbf{P 4 5 0}_{\mathrm{SCC}}$ were much weaker than their aromatase-inhibitory activities. These compounds may be regarded as selective aromatase inhibitors.
\end{abstract}

Key words 1,3,5-triazine; aromatase-inhibitory activity; CGS 16949A; P450 $\mathrm{SCC}$

Estrogen receptor-positive breast cancer in either premenopausal women or postmenopausal women is likely to be promoted by estrogen. ${ }^{1)}$ In postmenopausal women, the major pathway of estrogen biosynthesis is the peripheral conversion of androstenedione secreted by the adrenal cortex to estrone, for example, in the adipose tissue. This conversion is catalyzed by aromatase, which is a cytochrome P450-dependent monooxygenase. ${ }^{2)}$ Thus, aromatase inhibitors might be applicable as antitumor agents for estrogen-dependent breast cancer of postmenopausal women. With the aim of pharmacologically interfering with steroidogenesis, Griffiths et al. ${ }^{3)}$ proposed the use of aminoglutethimide (AG) combined with dexamethasone to suppress adrenal function. $A G$ is a potent inhibitor of the conversion of cholesterol to pregnenolone, ${ }^{4)}$ and it simultaneously acts as a nonsteroidal inhibitor of aromatase. ${ }^{5)}$

However, AG primarily acts as an inhibitor of cytochrome P450-catalyzed side chain cleavage of cholesterol ( $\mathrm{P} 450_{\text {sCC }}$ ), depleting corticosteroid production. ${ }^{6}$ ) The efforts of many groups to find selective aromatase inhibitors resulted in the discovery of CGS 16949A as a potent nonsteroidal aromatase inhibitor. ${ }^{7)}$

On the other hand, hexamethylmelamine (HMM), a 1,3,5-triazine derivative, which has been used as an antitumor agent to treat breast cancer $^{8)}$ and ovarian cancer, ${ }^{9)}$ does not show aromatase-inhibitory activity. Recently it has been suggested that an imidazole moiety is essential for aromatase-inhibitory activity, as in CGS 16949A or clotrimazole. ${ }^{10)}$ Therefore, imidazolyl-1,3,5triazine, which combines the two moieties, may be worth testing. ${ }^{11)}$ The aim of the present study was to seek novel aromatase inhibitors which are more potent and selective than CGS 16949A, by synthesizing a series of imidazolyl1,3,5-triazine derivatives, and examining their inhibitory activities towards aromatase and $\mathrm{P} 40_{\mathrm{ScC}}$.

Chemistry The desired 1,3,5-triazine derivatives I-IV were prepared by nucleophilic substitution reaction of azoles or aliphatic amines as shown in Chart 1.

Monochlorotriazine $\mathbf{1}$ and dichlorotriazine $\mathbf{2}$ were synthesized according to the method of Thurston et al. ${ }^{12)}$ However, the method was found to be inconvenient in that it yielded a mixture of $\mathbf{1}$ and $\mathbf{2}$. For the selective synthesis of either compound $\mathbf{1}$ or $\mathbf{2}$, care should be taken that the initial reaction temperature is kept at -15 to $-5^{\circ} \mathrm{C}$ for several hours using ethyleneglycol dimethyl ether as a reaction solvent for the synthesis of compound 2 , and at 0 to $10^{\circ} \mathrm{C}$ using acetone as a reaction solvent for compound 1. Furthermore, to avoid amine exchange reaction, the aliphatic amine should be employed in the first nucleophilic substitution reaction step. For example, when compound $\mathbf{3}$ was treated with morpholine for the synthesis of 14, the product obtained was not the desired compound 14, but 29. It seems that the morpholino group was changed to an imidazolyl group, which acted as a leaving group to afford 29.

Then, compound 2 was treated with amine and azole in the presence of base to afford $\mathbf{3}$ or $\mathbf{4}$ and compounds III, respectively. Because of the low activity of the monochlorotriazine for nucleophilic substitution reaction, the reactions of 1, 3 and 4 with azole were carried out at higher temperature than that used for the synthesis of 3

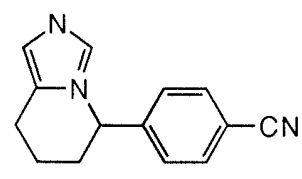

CGS 16949A

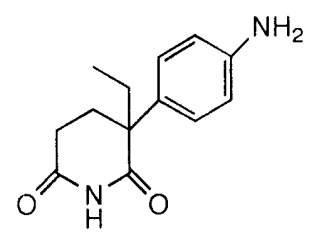

$A G$

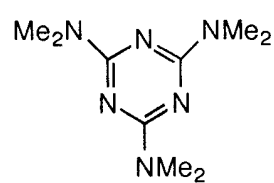

HMM

Fig. 1 


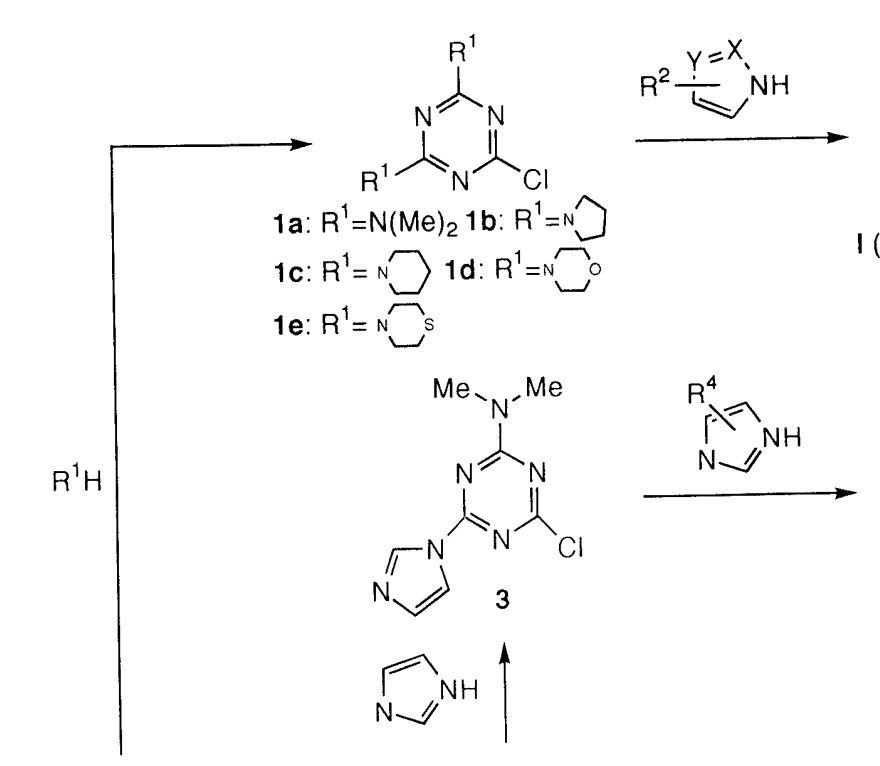

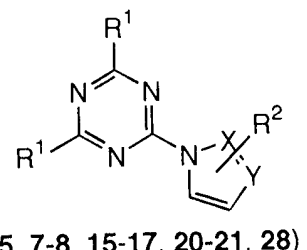

I (5, 7-8, 15-17, 20-21, 28)<smiles>Clc1nc(Cl)nc(Cl)n1</smiles>

Cyanuric chloride

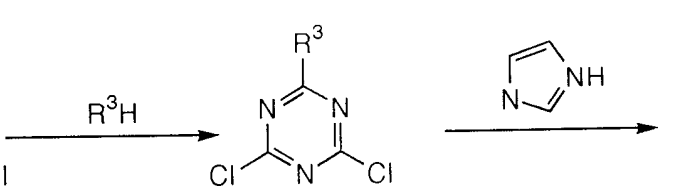

2a: $R^{3}=N(M e)_{2} 2 b: R^{3}=N O$ 2c: $R^{3}=N(M e) P h$<smiles>CPCPc1nc(P)nc(Cl)n1</smiles><smiles>c1c[nH]cn1</smiles>

4a: $R^{3}=\mathrm{N}(\mathrm{Me})_{2}, \mathrm{R}^{5}=\mathrm{NHMe}$ 4f: $R^{3}=$<smiles></smiles>

$R^{5}=N$

4g: $R^{3}=N$

4h: $R^{3}=N$

4c: $R^{3}=N(M e)_{2}, R^{5}=N$

4d: $R^{3}=\mathrm{N}(\mathrm{Me})_{2}, \mathrm{R}^{5}=\mathrm{N}$

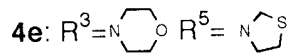

4i: $R^{3}=N$

4j: $R^{3}=N(M e) P h, R^{5}=N$<smiles></smiles>

II $(10,11)$<smiles>[B]c1nc(-n2ccnc2)nc(-n2ccnc2)n1</smiles>

III $(9,18)$<smiles>Pc1nc(P)nc(-n2ccnc2)n1</smiles>

IV $(6,12-14,22-27)$

\section{Chart 1}

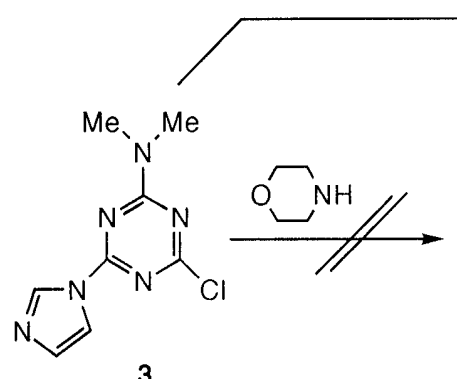<smiles>CN(C)c1nc(N2CCOCC2)nc(-n2ccnc2)n1</smiles>

14<smiles>CN(C)c1nc(N2CCOCC2)nc(N2CCOCC2)n1</smiles>

29

Chart 2

from dichlorotriazine 2, to afford I, II and IV, respectively.

Because substituted imidazoles, triazoles and pyrazoles are tautomeric, it is difficult to determine the structure of the product. To avoid this problem, 2-isopropylimidazole or 4,5-dimethylimidazole was employed as a starting material. In the case of the reaction of 1,2,3-triazole, a single product was obtained. The NMR spectra indicated that this compound is not 2-substituted, but is the 1substituted triazole derivative, because two protons in the triazole ring show two signals. ${ }^{13)}$

\section{Pharmacological Results and Discussion}

The compounds listed in Table 1 were evaluated for 
Table 1. Biological Properties of 1,3,5-Triazines

\begin{tabular}{|c|c|c|c|c|c|}
\hline Compd. & $\mathrm{R}^{1}$ & $\mathrm{R}^{2}$ & $\mathrm{R}^{3}$ & $\begin{array}{c}\text { Inhibition of aromatase } \\
\mathrm{IC}_{50}(\mathrm{M}) \text { or } \\
\text { inhibitory } \%(\mathrm{M})\end{array}$ & $\begin{array}{c}\text { Inhibition of } \mathrm{P} 450_{\mathrm{scc}} \\
\mathrm{IC}_{50}(\mathrm{M}) \text { or } \\
\text { inhibitory } \%(\mathrm{M})\end{array}$ \\
\hline 5 & $-\mathrm{NMe}_{2}$ & $-\mathrm{NMe}_{2}$ & & $4.1 \times 10^{-6}$ & $0 \%\left(1 \times 10^{-4}\right)$ \\
\hline 6 & $-\mathrm{NH}-\mathrm{Me}$ & $-\mathrm{NMe}_{2}$ & & $2.7 \%\left(1 \times 10^{-6}\right)$ & ND \\
\hline 7 & $-\mathrm{NMe}_{2}$ & $-\mathrm{NMe}_{2}$ & & $0 \%\left(1 \times 10^{-4}\right)$ & ND \\
\hline 8 & $-\mathrm{NMe}_{2}$ & $-\mathrm{NMe}_{2}$ & & $0 \%\left(1 \times 10^{-4}\right)$ & ND \\
\hline 9 & $-\mathrm{NMe}_{2}$ & & & $5.5 \times 10^{-7}$ & $8 \times 10^{-5}$ \\
\hline 10 & $-\mathrm{NMe}_{2}$ & & & $4.3 \times 10^{-6}$ & $30 \%\left(1 \times 10^{-4}\right)$ \\
\hline 11 & $-\mathrm{NMe}_{2}$ & & & $2.0 \times 10^{-7}$ & $3 \%\left(1 \times 10^{-4}\right)$ \\
\hline 12 & $-\mathrm{NMe}_{2}$ & & & $1.3 \times 10^{-6}$ & $14 \%\left(1 \times 10^{-4}\right)$ \\
\hline 13 & $-\mathrm{NMe}_{2}$ & & & $7.7 \times 10^{-7}$ & $26 \%\left(1 \times 10^{-4}\right)$ \\
\hline 14 & $-\mathrm{NMe}_{2}$ & & & $1.0 \times 10^{-8}$ & $1 \%\left(1 \times 10^{-4}\right)$ \\
\hline 15 & & & & $3.1 \times 10^{-7}$ & $40 \%\left(1 \times 10^{-4}\right)$ \\
\hline 16 & & & & $3.1 \times 10^{-7}$ & $30 \%\left(1 \times 10^{-4}\right)$ \\
\hline 17 & & & & $5.3 \times 10^{-9}$ & $1 \%\left(1 \times 10^{-4}\right)$ \\
\hline 18 & & & & $2.1 \times 10^{-8}$ & $0 \%\left(1 \times 10^{-4}\right)$ \\
\hline 19 & & & & $0 \%\left(1 \times 10^{-6}\right)$ & ND \\
\hline 20 & & & & $3.8 \%\left(1 \times 10^{-6}\right)$ & ND \\
\hline 21 & & & & $0 \%\left(1 \times 10^{-4}\right)$ & ND \\
\hline 22 & & & & $3.1 \times 10^{-8}$ & $45 \%\left(1 \times 10^{-4}\right)$ \\
\hline 23 & & & & $6.6 \times 10^{-9}$ & $7.2 \times 10^{-5}$ \\
\hline 24 & & & & $1.7 \times 10^{-8}$ & $36.8 \%\left(2 \times 10^{-5}\right)$ \\
\hline 25 & & & & $2.4 \times 10^{-8}$ & $28 \%\left(5 \times 10^{-5}\right)$ \\
\hline 26 & & & & $9.0 \times 10^{-9}$ & $2.2 \times 10^{-5}$ \\
\hline 27 & & & & $6.7 \times 10^{-9}$ & $3.1 \times 10^{-5}$ \\
\hline
\end{tabular}


Table 1. (continued)

\begin{tabular}{|c|c|c|c|c|c|}
\hline Compd. & $\mathrm{R}^{1}$ & $\mathrm{R}^{2}$ & $\mathrm{R}^{3}$ & $\begin{array}{c}\text { Inhibition of aromatase } \\
\mathrm{IC}_{50}(\mathrm{M}) \text { or } \\
\text { inhibitory } \%(\mathrm{M})\end{array}$ & $\begin{array}{c}\text { Inhibition of } \mathrm{P} 450_{\text {sce }} \\
\mathrm{IC}_{50}(\mathrm{M}) \text { or } \\
\text { inhibitory } \%(\mathrm{M})\end{array}$ \\
\hline 28 & & & & $5.1 \times 10^{-9}$ & $0 \%\left(1 \times 10^{-6}\right)$ \\
\hline $\begin{array}{c}\mathrm{HMM} \\
\mathrm{AG}\end{array}$ & $\begin{array}{r}-\mathrm{NMe}_{2} \\
\text { CGS-16949A }\end{array}$ & $-\mathrm{NMe}_{2}$ & $-\mathrm{NMe}_{2}$ & $\begin{array}{r}5.4 \%\left(1 \times 10^{-4}\right) \\
3.8 \times 10^{-5} \\
1.7 \times 10^{-8}\end{array}$ & $\begin{array}{l}\text { ND } \\
6.6 \times 10^{-5} \\
6 \times 10^{-5}\end{array}$ \\
\hline
\end{tabular}

ND: not determined.

inhibitory activity against aromatase from human placental microsomes and for inhibitory activity against $\mathrm{P}^{450} 0_{\text {SCC }}$ from pig adrenal mitochondria. Almost all the compounds, which have one or two imidazolyl group as substituents on the 1,3,5-triazine moiety, showed potent aromatase-inhibitory activity. A remarkable decrease in potency was observed upon replacing the imidazolyl group of $\mathbf{1 7}$ with other functional groups, such as a morpholino group (19), pyrazolyl group (20), or triazolyl group (21). Moreover, replacing the dimethylamino group of $\mathbf{5}$ with a methylamino group resulted in a remarkable decrease in the aromatase-inhibitory activity (6). These results suggest that the imidazolyl group and the tertiary amino group are necessary for potent aromatase-inhibitory activity. However, the substitution of the 2-position on the imidazole ring with an isopropyl group as in compound 7 or the substitution of the 4- and 5-position on the imidazole ring with a methyl group as in compound $\mathbf{8}$ caused a reduction of the activity. This result suggests that a bulky isopropyl group or methyl group, which prevents the access of the imidazole ring to the active site of the enzyme, is unfavorable for the inhibitory activity. Compounds 17, 18 and $22-27$ all have a morpholino and an imidazolyl group, and showed high aromatase-inhibitory activity. Compound $\mathbf{2 8}$, in which the morpholino group was replaced with a thiomorpholino group also showed high aromatase-inhibitory activity. Compounds 17, 23 and 26-28 showed two- or three-fold higher activity than that of the reference compound, CGS $16949 \mathrm{~A}$. In order to understand whether these compounds have potent antitumor activity against estrogen-dependent breast cancer, we examined the effect of these compounds in suppressing uterine weight in rats. The results will be reported in a separate paper.

\section{Experimental}

Melting points were determined on a Yamato melting point apparatus Model Mp-21, and are uncorrected. Mass spectra (MS) were recorded on a JEOL GX102 spectrometer. ${ }^{1} \mathrm{H}-\mathrm{NMR}$ spectra were taken at $270 \mathrm{MHz}$ with a JEOL spectrometer with tetramethylsilane as an internal standard. Elemental analysis was performed using a Yanagimoto MT-2 analyzer. Column chromatography was carried out on silica gel (Wakogel C-200). CGS 16949A, ${ }^{14)} \mathrm{HMM}^{15)}$ and $19^{16)}$ were prepared according to the literature.

General Procedure for Preparation of Monochloro-1,3,5-triazines 1 from Cyanuric Chloride Synthesis of 2-Chloro-4,6-bis(dimethylamino)1,3,5-triazine (1a): An aqueous solution of dimethylamine ( $50 \%$ solution, $19.0 \mathrm{ml}, 211 \mathrm{mmol}$ ) was slowly added dropwise to a solution of cyanuric chloride $(9.22 \mathrm{~g}, 50.0 \mathrm{mmol})$ in $\mathrm{Me}_{2} \mathrm{CO}(100 \mathrm{ml})$ at 0 to $10^{\circ} \mathrm{C}$. The reaction mixture was stirred at $0^{\circ} \mathrm{C}$ for $2 \mathrm{~h}$ and then at room temperature for $18 \mathrm{~h}$. After removal of the solvent, the residue was extracted with
$\mathrm{CH}_{2} \mathrm{Cl}_{2}$. The extract was washed with brine, dried over $\mathrm{MgSO}_{4}$ and concentrated. The residue was column-chromatographed on silica gel with hexane-ethyl acetate (AcOEt) $(8: 2)$ to give $1 \mathrm{a}(9.9 \mathrm{~g})$ in $98 \%$ yield. Colorless crystals, mp 89-91 ${ }^{\circ} \mathrm{C} .{ }^{1} \mathrm{H}-\mathrm{NMR}\left(\mathrm{CDCl}_{3}\right) \delta: 3.13(12 \mathrm{H}, \mathrm{s})$. MS $m / z: 201\left(\mathbf{M}^{+}\right)$.

Compound $\mathbf{1 b}-\mathbf{e}$ were similarly prepared from cyanuric chloride.

2-Chloro-4,6-dipyrrolidino-1,3,5-triazine (1b): $25 \%$ yield. Colorless crystals, mp $112-115^{\circ} \mathrm{C} .{ }^{1} \mathrm{H}-\mathrm{NMR}\left(\mathrm{CDCl}_{3}\right) \delta: 1.9-2.0(8 \mathrm{H}, \mathrm{m})$, 3.5-3.7 (8H, m). MS $m / z: 253\left(\mathrm{M}^{+}\right)$.

2-Chloro-4,6-dipiperidino-1,3,5-triazine (1c): $75 \%$ yield. Colorless crystals, mp $114-117^{\circ} \mathrm{C} .{ }^{1} \mathrm{H}-\mathrm{NMR}\left(\mathrm{CDCl}_{3}\right) \delta: 1.5-1.7(12 \mathrm{H}, \mathrm{m}), 3.73$ $(8 \mathrm{H}, \mathrm{t}, J=5 \mathrm{~Hz})$. MS $m / z: 281\left(\mathrm{M}^{+}\right)$.

2-Chloro-4,6-dimorpholino-1,3,5-triazine (1d): $91 \%$ yield. Colorless crystals, $\mathrm{mp} 173-174^{\circ} \mathrm{C} .{ }^{1} \mathrm{H}-\mathrm{NMR}\left(\mathrm{CDCl}_{3}\right) \delta: 3.6-3.8(16 \mathrm{H}, \mathrm{m})$. MS $m / z: 285\left(\mathrm{M}^{+}\right)$.

2-Chloro-4,6-dithiomorpholino-1,3,5-triazine (1e): 12\% yield. Colorless crystals, mp $192-194{ }^{\circ} \mathrm{C} .{ }^{1} \mathrm{H}-\mathrm{NMR}\left(\mathrm{CDCl}_{3}\right) \delta: 2.6-2.7(8 \mathrm{H}, \mathrm{m})$, $4.07(8 \mathrm{H}, \mathrm{brs})$. MS $m / z: 317\left(\mathrm{M}^{+}\right)$.

These were used for the next reaction without further purification.

General Procedure for Preparation of Dichloro-1,3,5-triazines 2 Synthesis of 2,4-Dichloro-6-dimethylamino-1,3,5-triazine (2a): An aqueous solution of dimethylamine ( $50 \%$ solution, $10.8 \mathrm{ml}, 120 \mathrm{mmol}$ ) was slowly added dropwise to a solution of cyanuric chloride $(11.1 \mathrm{~g}, 60.2 \mathrm{mmol})$ in ethyleneglycol dimethyl ether $(130 \mathrm{ml})$ at -15 to $-5^{\circ} \mathrm{C}$. The reaction mixture was stirred at $-15^{\circ} \mathrm{C}$ for $2 \mathrm{~h}$ and then at room temperature for $20 \mathrm{~h}$. After removal of the solvent, the residue was extracted with $\mathrm{CH}_{2} \mathrm{Cl}_{2}$. The extract was washed with brine, dried over $\mathrm{MgSO}_{4}$ and concentrated to give $2 \mathrm{a}(11.0 \mathrm{~g})$ in $95 \%$ yield. Colorless crystals, $\mathrm{mp} 122-124^{\circ} \mathrm{C}$. ${ }^{1} \mathrm{H}-\mathrm{NMR}\left(\mathrm{CDCl}_{3}\right) \delta: 3.13(6 \mathrm{H}, \mathrm{s})$. MS $m / z: 192\left(\mathrm{M}^{+}\right)$.

Compounds $\mathbf{2 b}$ and $\mathbf{2 c}$ were similarly prepared from cyanuric chloride.

2,4-Dichloro-6-morpholino-1,3,5-triazine (2b): 63\% yield. Colorless crystals, mp $162-164^{\circ} \mathrm{C} .{ }^{1} \mathrm{H}-\mathrm{NMR}\left(\mathrm{CDCl}_{3}\right) \delta: 3.6-3.8(8 \mathrm{H}, \mathrm{m}) . \mathrm{MS}$ $m / z: 234\left(\mathbf{M}^{+}\right)$.

2,4-Dichloro-6-( $N$-methyl- $N$-phenylamino)-1,3,5-triazine (2c): $61 \%$ yield. Colorless crystals, mp $131-133{ }^{\circ} \mathrm{C} .{ }^{1} \mathrm{H}-\mathrm{NMR}\left(\mathrm{CDCl}_{3}\right) \delta: 3.55$ $(3 \mathrm{H}, \mathrm{s}), 7.2-7.5(5 \mathrm{H}, \mathrm{m})$. MS $m / z: 254\left(\mathrm{M}^{+}\right)$.

These were used for the next reaction without further purification.

2-Chloro-4-dimethylamino-6-(1-imidazolyl)-1,3,5-triazine (3): Imidazole $(0.681 \mathrm{~g}, 10.0 \mathrm{mmol})$ and $\mathrm{K}_{2} \mathrm{CO}_{3}(1.38 \mathrm{~g}, 10.0 \mathrm{mmol})$ were added to a solution of $2 \mathrm{a}(1.93 \mathrm{~g}, 10.0 \mathrm{mmol})$ in $N, N$-dimethylformamide (DMF) $(8 \mathrm{ml})$ at room temperature. The reaction mixture was stirred at room temperature for $17 \mathrm{~h}$, then evaporated in vacuo, and the residue was extracted with AcOEt. The extract was washed with brine, dried over $\mathrm{MgSO}_{4}$ and evaporated in vacuo. The residue was column-chromatographed on silica gel with AcOEt-methanol (MeOH) $(95: 5)$ to give 3 $(0.87 \mathrm{~g})$ in $39 \%$ yield. Colorless crystals, mp $87-90{ }^{\circ} \mathrm{C} .{ }^{1} \mathrm{H}-\mathrm{NMR}$ $\left(\mathrm{CDCl}_{3}\right) \delta: 3.27(6 \mathrm{H}, \mathrm{s}), 7.13(1 \mathrm{H}, \mathrm{s}), 7.78(1 \mathrm{H}, \mathrm{s}), 8.54(1 \mathrm{H}, \mathrm{m}) . \mathrm{MS}$ $m / z: 224\left(\mathrm{M}^{+}\right)$.

This was used for the next reaction without further purification.

General Procedure for Preparation of Monochloro-1,3,5-triazines 4 Synthesis of 2-Chloro-4-dimethylamino-6-methylamino-1,3,5-triazine (4a): An aqueous solution of methylamine $(40 \%$ solution, $0.16 \mathrm{ml}$, $2.0 \mathrm{mmol})$ was slowly added dropwise to a solution of $2 \mathrm{a}(0.39 \mathrm{~g}$, $2.0 \mathrm{mmol})$ in DMF $(6 \mathrm{ml})$ at -5 to $0{ }^{\circ} \mathrm{C}$ in the presence of $\mathrm{K}_{2} \mathrm{CO}_{3}(0.56 \mathrm{~g}$, $4.1 \mathrm{mmol})$ as a hydrogen chloride scavenger. The resulting reaction mixture was stirred at $-5^{\circ} \mathrm{C}$ for $2 \mathrm{~h}$ and then at room temperature for $20 \mathrm{~h}$. After removal of the solvent, the residue was extracted with AcOEt. The extract was washed with brine, dried over $\mathrm{MgSO}_{4}$ and concentrated. The residue was column-chromatographed on silica gel with AcOEt $(7: 3)$ 
to give the crude product $4 \mathrm{a}(0.18 \mathrm{~g})$ in $47 \%$ yield. Colorless crystals, $\mathrm{mp}$ 206-208 ${ }^{\circ} \mathrm{C} .{ }^{1} \mathrm{H}-\mathrm{NMR}\left(\mathrm{CDCl}_{3}\right) \delta: 2.98(3 \mathrm{H}, \mathrm{d}, J=5 \mathrm{~Hz}), 3.16(6 \mathrm{H}, \mathrm{s})$, $6.27\left(1 \mathrm{H}\right.$, br s). MS $m / z: 187\left(\mathrm{M}^{+}\right)$.

Compounds $4 \mathrm{~b}-\mathrm{j}$ were similarly prepared from dichloro-1,3,5triazine 2.

2-Chloro-4-dimethylamino-6-pyrrolidino-1,3,5-triazine (4b): $45 \%$ yield. Colorless oil. ${ }^{1} \mathrm{H}-\mathrm{NMR}\left(\mathrm{CDCl}_{3}\right) \delta: 1.9-2.0(4 \mathrm{H}, \mathrm{m}), 3.13(6 \mathrm{H}$, s), 3.5-3.6 (4H, m). MS $m / z: 227\left(\mathrm{M}^{+}\right)$.

2-Chloro-4-dimethylamino-6-piperidino-1,3,5-triazine (4c): $81 \%$ yield. Colorless crystals, mp $68-72{ }^{\circ} \mathrm{C} .{ }^{1} \mathrm{H}-\mathrm{NMR}\left(\mathrm{CDCl}_{3}\right) \delta: 1.5-1.8(6 \mathrm{H}$, $\mathrm{m}), 3.13(6 \mathrm{H}, \mathrm{s}), 3.74(4 \mathrm{H}, \mathrm{t}, J=5 \mathrm{~Hz})$. MS $m / z: 241\left(\mathrm{M}^{+}\right)$.

2-Chloro-4-dimethylamino-6-morpholino-1,3,5-triazine (4d): $55 \%$ yield. Colorless crystals, mp $96-98^{\circ} \mathrm{C} .{ }^{1} \mathrm{H}-\mathrm{NMR}\left(\mathrm{CDCl}_{3}\right) \delta: 3.13(6 \mathrm{H}$, s), $3.6-3.8(8 \mathrm{H}, \mathrm{m})$. MS $m / z: 243\left(\mathrm{M}^{+}\right)$.

2-Chloro-4-morpholino-6-thiazolidino-1,3,5-triazine (4e): $97 \%$ yield. Colorless crystals, mp 137-139 ${ }^{\circ} \mathrm{C} .{ }^{1} \mathrm{H}-\mathrm{NMR}\left(\mathrm{CDCl}_{3}\right) \delta: 3.07(2 \mathrm{H}, \mathrm{t}$, $J=6 \mathrm{~Hz}), 3.6-4.0(10 \mathrm{H}, \mathrm{m}), 4.66(1 \mathrm{H}, \mathrm{s}), 4.71(1 \mathrm{H}, \mathrm{s}) . \mathrm{MS} m / z: 287$ $\left(\mathrm{M}^{+}\right)$.

2-Chloro-4-(cis-2,6-dimethylmorpholino)-6-morpholino-1,3,5-triazine (4f): $73 \%$ yield. Colorless crystals, mp $185-187^{\circ} \mathrm{C} .{ }^{1} \mathrm{H}-\mathrm{NMR}\left(\mathrm{CDCl}_{3}\right)$ $\delta: 1.24(6 \mathrm{H}, \mathrm{d}, J=6.0 \mathrm{~Hz}), 2.57(2 \mathrm{H}, \mathrm{t}, J=12 \mathrm{~Hz}), 3.5-3.9(10 \mathrm{H}, \mathrm{m})$, $4.4-4.6(2 \mathrm{H}, \mathrm{m})$. MS $m / z: 313\left(\mathrm{M}^{+}\right)$.

2-(4-Benzylpiperazino)-4-chloro-6-morpholino-1,3,5-triazine (4g): $88 \%$ yield. Colorless oil. ${ }^{1} \mathrm{H}-\mathrm{NMR}\left(\mathrm{CDCl}_{3}\right) \delta: 2.47(4 \mathrm{H}, \mathrm{br} \mathrm{s}), 3.56(2 \mathrm{H}$,

Table 2. Properties of the Compounds in Table 1

\begin{tabular}{|c|c|c|c|c|c|c|c|c|}
\hline \multirow{2}{*}{ Compd. } & \multirow{2}{*}{$\begin{array}{l}\text { Yield } \\
(\%)\end{array}$} & \multirow{2}{*}{$\begin{array}{l}\mathrm{mp} \\
\left({ }^{\circ} \mathrm{C}\right)\end{array}$} & \multirow{2}{*}{ Formula } & \multicolumn{3}{|c|}{$\begin{array}{l}\text { Analysis (\%) } \\
\text { Calcd (Found) }\end{array}$} & \multirow{2}{*}{$\begin{array}{c}\mathrm{MS} m / z \\
{\left[\mathrm{M}^{+}\right]}\end{array}$} & \multirow{2}{*}{${ }^{1} \mathrm{H}-\mathrm{NMR}\left(\mathrm{CDCl}_{3}\right) \delta$} \\
\hline & & & & $\mathrm{C}$ & $\mathrm{H}$ & $\mathrm{N}$ & & \\
\hline 5 & 80 & $129-131$ & $\mathrm{C}_{10} \mathrm{H}_{15} \mathrm{~N}_{7}$ & $\begin{array}{r}51.49 \\
(51.63\end{array}$ & $\begin{array}{l}6.48 \\
6.54\end{array}$ & $\begin{array}{l}42.03 \\
42.11)\end{array}$ & 233 & $\begin{array}{l}3.17(12 \mathrm{H}, \mathrm{s}), 7.08(1 \mathrm{H}, \mathrm{d}, J=1 \mathrm{~Hz}), 7.80(1 \mathrm{H}, \mathrm{d}, J=1 \mathrm{~Hz}), 8.53 \\
(1 \mathrm{H}, \mathrm{s})\end{array}$ \\
\hline 6 & 91 & $164-166$ & $\mathrm{C}_{9} \mathrm{H}_{13} \mathrm{~N}_{7}$ & $\begin{array}{r}49.30 \\
(49.32\end{array}$ & $\begin{array}{l}5.98 \\
6.08\end{array}$ & $\begin{array}{l}44.72 \\
44.60)\end{array}$ & 219 & $\begin{array}{l}3.01(3 \mathrm{H}, \mathrm{d}, J=5 \mathrm{~Hz}), 3.20(6 \mathrm{H}, \mathrm{s}), 5.17(1 \mathrm{H}, \mathrm{brs}), 7.08(1 \mathrm{H}, \mathrm{s}), \\
7.75(1 \mathrm{H}, \mathrm{s}), 8.51(1 \mathrm{H}, \mathrm{s})\end{array}$ \\
\hline 7 & 50 & $130-131$ & $\mathrm{C}_{13} \mathrm{H}_{21} \mathrm{~N}_{7}$ & $\begin{array}{r}56.71 \\
(56.53\end{array}$ & $\begin{array}{l}7.69 \\
7.67\end{array}$ & $\begin{array}{l}35.61 \\
35.53)\end{array}$ & 275 & $\begin{array}{l}1.36(6 \mathrm{H}, \mathrm{d}, J=7 \mathrm{~Hz}), 3.16(12 \mathrm{H}, \mathrm{s}), 4.18(1 \mathrm{H}, \text { septet, } J=7 \mathrm{~Hz}) \\
6.91(1 \mathrm{H}, \mathrm{d}, J=2 \mathrm{~Hz}), 7.80(1 \mathrm{H}, \mathrm{d}, J=2 \mathrm{~Hz})\end{array}$ \\
\hline 8 & 42 & $135-137$ & $\mathrm{C}_{12} \mathrm{H}_{19} \mathrm{~N}_{7}$ & $\begin{array}{r}55.15 \\
(55.01\end{array}$ & $\begin{array}{l}7.33 \\
7.33\end{array}$ & $\begin{array}{l}37.52 \\
37.40)\end{array}$ & 261 & $2.18(3 \mathrm{H}, \mathrm{s}), 2.50(3 \mathrm{H}, \mathrm{s}), 3.16(12 \mathrm{H}, \mathrm{s}), 8.43(1 \mathrm{H}, \mathrm{s})$ \\
\hline 9 & 65 & $172-174$ & $\mathrm{C}_{11} \mathrm{H}_{12} \mathrm{~N}_{8}$ & $\begin{array}{r}51.56 \\
(51.69\end{array}$ & $\begin{array}{l}4.72 \\
4.77\end{array}$ & $\begin{array}{l}43.72 \\
43.70)\end{array}$ & 256 & $3.31(6 \mathrm{H}, \mathrm{s}), 7.16(2 \mathrm{H}, \mathrm{s}), 7.83(2 \mathrm{H}, \mathrm{s}), 8.59(2 \mathrm{H}, \mathrm{s})$ \\
\hline 10 & 60 & $130-131$ & $\mathrm{C}_{14} \mathrm{H}_{18} \mathrm{~N}_{8}$ & $\begin{array}{r}56.36 \\
(56.12\end{array}$ & $\begin{array}{l}6.08 \\
6.05\end{array}$ & $\begin{array}{l}37.56 \\
37.43)\end{array}$ & 298 & $\begin{array}{l}1.43(6 \mathrm{H}, \mathrm{d}, J=7 \mathrm{~Hz}), 3.30(3 \mathrm{H}, \mathrm{s}), 3.32(3 \mathrm{H}, \mathrm{s}), 4.09(1 \mathrm{H} \\
\text { septet, } J=7 \mathrm{~Hz}), 6.99(1 \mathrm{H}, \mathrm{s}), 7.17(1 \mathrm{H}, \mathrm{s}), 7.82(1 \mathrm{H}, \mathrm{s}), 7.87 \\
(1 \mathrm{H}, \mathrm{s}), 8.57(1 \mathrm{H}, \mathrm{s})\end{array}$ \\
\hline 11 & 88 & $192-194$ & $\mathrm{C}_{13} \mathrm{H}_{16} \mathrm{~N}_{8}$ & $\begin{array}{r}54.92 \\
(54.90\end{array}$ & $\begin{array}{l}5.67 \\
5.57\end{array}$ & $\begin{array}{l}39.41 \\
39.54)\end{array}$ & 284 & $\begin{array}{l}2.21(3 \mathrm{H}, \mathrm{s}), 2.54(3 \mathrm{H}, \mathrm{s}), 3.27(3 \mathrm{H}, \mathrm{s}), 3.29(3 \mathrm{H}, \mathrm{s}), 7.15(1 \mathrm{H}, \\
\mathrm{s}), 7.80(1 \mathrm{H}, \mathrm{s}), 8.50(1 \mathrm{H}, \mathrm{s}), 8.56(1 \mathrm{H}, \mathrm{s})\end{array}$ \\
\hline 12 & 63 & $101-103$ & $\mathrm{C}_{12} \mathrm{H}_{17} \mathrm{~N}_{7}$ & $\begin{array}{r}55.58 \\
(55.48\end{array}$ & $\begin{array}{l}6.61 \\
6.60\end{array}$ & $\begin{array}{l}37.81 \\
37.65)\end{array}$ & 259 & $\begin{array}{l}1.9-2.0(4 \mathrm{H}, \mathrm{m}), 3.17(6 \mathrm{H}, \mathrm{s}), 3.5-3.7(4 \mathrm{H}, \mathrm{m}), 7.07(1 \mathrm{H}, \mathrm{s}), \\
7.80(\mathrm{lH}, \mathrm{s}), 8.53(1 \mathrm{H}, \mathrm{s})\end{array}$ \\
\hline 13 & 65 & $98-99$ & $\mathrm{C}_{13} \mathrm{H}_{19} \mathrm{~N}_{7}$ & $\begin{array}{r}57.12 \\
(56.96\end{array}$ & $\begin{array}{l}7.01 \\
7.07\end{array}$ & $\begin{array}{l}35.87 \\
35.64)\end{array}$ & 273 & $\begin{array}{l}1.5-1.8(6 \mathrm{H}, \mathrm{m}), 3.17(6 \mathrm{H}, \mathrm{s}), 3.80(4 \mathrm{H}, \mathrm{t}, J=5 \mathrm{~Hz}), 7.07(1 \mathrm{H} \\
\mathrm{s}), 7.79(1 \mathrm{H}, \mathrm{s}), 8.52(1 \mathrm{H}, \mathrm{s})\end{array}$ \\
\hline 14 & 92 & $156-158$ & $\mathrm{C}_{12} \mathrm{H}_{17} \mathrm{~N}_{7} \mathrm{O}$ & $\begin{array}{r}52.35 \\
(52.31\end{array}$ & $\begin{array}{l}6.22 \\
6.17\end{array}$ & $\begin{array}{l}35.61 \\
35.41)\end{array}$ & 275 & $\begin{array}{l}3.15(3 \mathrm{H}, \mathrm{s}), 3.20(3 \mathrm{H}, \mathrm{s}), 3.74(4 \mathrm{H}, \mathrm{t}, J=4 \mathrm{~Hz}), 3.85(4 \mathrm{H}, \mathrm{t} \\
J=4 \mathrm{~Hz}), 7.08(1 \mathrm{H}, \mathrm{s}), 7.77(1 \mathrm{H}, \mathrm{s}), 8.51(1 \mathrm{H}, \mathrm{s})\end{array}$ \\
\hline 15 & 87 & $162-163$ & $\mathrm{C}_{14} \mathrm{H}_{19} \mathrm{~N}_{7}$ & $\begin{array}{r}58.93 \\
(58.72\end{array}$ & $\begin{array}{l}6.71 \\
6.63\end{array}$ & $\begin{array}{l}34.36 \\
34.25)\end{array}$ & 285 & $\begin{array}{l}1.9-2.0(8 \mathrm{H}, \mathrm{m}), 3.5-3.7(8 \mathrm{H}, \mathrm{m}), 7.07(1 \mathrm{H}, \mathrm{s}), 7.80(1 \mathrm{H}, \mathrm{s}), \\
8.53(1 \mathrm{H}, \mathrm{s})\end{array}$ \\
\hline 16 & 96 & $154-156$ & $\mathrm{C}_{16} \mathrm{H}_{23} \mathrm{~N}_{7}$ & $\begin{array}{r}61.32 \\
(61.38\end{array}$ & $\begin{array}{l}7.40 \\
7.29\end{array}$ & $\begin{array}{l}31.28 \\
31.32)\end{array}$ & 313 & $\begin{array}{l}1.3-1.7(12 \mathrm{H}, \mathrm{m}), 3.71(8 \mathrm{H}, \mathrm{brs}), 7.00(1 \mathrm{H}, \mathrm{s}), 7.71(1 \mathrm{H}, \mathrm{s}) \\
8.44(1 \mathrm{H}, \mathrm{s})\end{array}$ \\
\hline 17 & 88 & $248-249$ & $\mathrm{C}_{14} \mathrm{H}_{19} \mathrm{~N}_{7} \mathrm{O}_{2}$ & $\begin{array}{r}52.99 \\
(52.97\end{array}$ & $\begin{array}{l}6.03 \\
6.00\end{array}$ & $\begin{array}{l}30.90 \\
30.64)\end{array}$ & 317 & $\begin{array}{l}3.74(8 \mathrm{H}, \mathrm{t}, J=5 \mathrm{~Hz}), 3.85(8 \mathrm{H}, \mathrm{br} \mathrm{s}), 7.09(1 \mathrm{H}, \mathrm{s}), 7.75(1 \mathrm{H}, \mathrm{s}) \text {, } \\
8.49(1 \mathrm{H}, \mathrm{s})\end{array}$ \\
\hline 18 & 33 & $268-270$ & $\mathrm{C}_{13} \mathrm{H}_{14} \mathrm{~N}_{8} \mathrm{O}$ & $\begin{array}{r}52.34 \\
(52.14\end{array}$ & $\begin{array}{l}4.73 \\
4.81\end{array}$ & $\begin{array}{l}37.56 \\
37.46)\end{array}$ & 298 & $\begin{array}{l}3.82(4 \mathrm{H}, \mathrm{t}, J=4 \mathrm{~Hz}), 3.99(4 \mathrm{H}, \mathrm{t}, J=4 \mathrm{~Hz}), 7.17(2 \mathrm{H}, \mathrm{s}), 7.82 \\
(2 \mathrm{H}, \mathrm{s}), 8.58(2 \mathrm{H}, \mathrm{s})\end{array}$ \\
\hline 20 & 71 & $220-221$ & $\mathrm{C}_{14} \mathrm{H}_{19} \mathrm{~N}_{7} \mathrm{O}_{2}$ & $\begin{array}{r}52.99 \\
(52.75\end{array}$ & $\begin{array}{l}6.03 \\
6.10\end{array}$ & $\begin{array}{l}30.90 \\
30.87)\end{array}$ & 317 & $\begin{array}{l}3.75(8 \mathrm{H}, \mathrm{t}, J=5 \mathrm{~Hz}), 3.8-3.9(8 \mathrm{H}, \mathrm{m}), 6.42(1 \mathrm{H}, \mathrm{dd}, J=1, \\
2 \mathrm{~Hz}), 7.79(1 \mathrm{H}, \mathrm{d}, J=1 \mathrm{~Hz}), 8.51(1 \mathrm{H}, \mathrm{d}, J=2 \mathrm{~Hz})\end{array}$ \\
\hline 21 & 43 & $239-240$ & $\mathrm{C}_{13} \mathrm{H}_{18} \mathrm{~N}_{8} \mathrm{O}_{2}$ & $\begin{array}{r}49.05 \\
(49.22\end{array}$ & $\begin{array}{l}5.70 \\
5.66\end{array}$ & $\begin{array}{l}35.20 \\
35.06)\end{array}$ & 318 & $\begin{array}{l}3.76(8 \mathrm{H}, \mathrm{t}, J=5 \mathrm{~Hz}), 3.8-3.9(8 \mathrm{H}, \mathrm{m}), 7.78(1 \mathrm{H}, \mathrm{d}, J=1 \mathrm{~Hz}) \\
8.48(1 \mathrm{H}, \mathrm{d}, J=1 \mathrm{~Hz})\end{array}$ \\
\hline 22 & 73 & $165-167$ & $\mathrm{C}_{16} \mathrm{H}_{23} \mathrm{~N}_{7} \mathrm{O}_{2}$ & $\begin{array}{r}55.64 \\
(55.49\end{array}$ & $\begin{array}{l}6.71 \\
6.71\end{array}$ & $\begin{array}{l}28.39 \\
28.36)\end{array}$ & 345 & $\begin{array}{l}1.26(6 \mathrm{H}, \mathrm{d}, J=6 \mathrm{~Hz}), 2.5-2.7(2 \mathrm{H}, \mathrm{m}), 3.5-3.9(10 \mathrm{H}, \mathrm{m}) \\
4.5-4.7(2 \mathrm{H}, \mathrm{m}), 7.09(1 \mathrm{H}, \mathrm{t}, J=1 \mathrm{~Hz}), 7.76(1 \mathrm{H}, \mathrm{t}, J=1 \mathrm{~Hz}) \\
8.49(1 \mathrm{H}, \mathrm{t}, J=1 \mathrm{~Hz})\end{array}$ \\
\hline 23 & 89 & $185--187$ & $\mathrm{C}_{13} \mathrm{H}_{17} \mathrm{~N}_{7} \mathrm{OS}$ & $\begin{array}{r}48.89 \\
(49.12\end{array}$ & $\begin{array}{l}5.37 \\
5.41\end{array}$ & $\begin{array}{l}30.70 \\
30.47)\end{array}$ & 319 & $\begin{array}{l}3.10(2 \mathrm{H}, \mathrm{t}, J=7 \mathrm{~Hz}), 3.74(4 \mathrm{H}, \mathrm{t}, J=4 \mathrm{~Hz}), 3.85(4 \mathrm{H}, \mathrm{br} \mathrm{s}) \\
3.9-4.0(2 \mathrm{H}, \mathrm{m}), 4.74(2 \mathrm{H}, \mathrm{brs}), 7.09(1 \mathrm{H}, \mathrm{s}), 7.77(1 \mathrm{H}, \mathrm{s}) \\
8.50(1 \mathrm{H}, \mathrm{s})\end{array}$ \\
\hline 24 & 99 & $207-209$ & $\mathrm{C}_{21} \mathrm{H}_{26} \mathrm{~N}_{8} \mathrm{O}$ & $\begin{array}{r}62.05 \\
(62.08\end{array}$ & $\begin{array}{l}6.45 \\
6.54\end{array}$ & $\begin{array}{l}27.57 \\
27.35)\end{array}$ & 406 & $\begin{array}{l}2.49(4 \mathrm{H}, \mathrm{brs}), 3.55(2 \mathrm{H}, \mathrm{br} \mathrm{s}), 3.73(4 \mathrm{H}, \mathrm{t}, J=5 \mathrm{~Hz}), 3.83(8 \mathrm{H}, \\
\mathrm{brs}), 7.07(1 \mathrm{H}, \mathrm{dd}, J=1,2 \mathrm{~Hz}), 7.25-7.34(5 \mathrm{H}, \mathrm{m}), 7.74(1 \mathrm{H}, \\
\text { d, } J=2 \mathrm{~Hz}), 8.48(1 \mathrm{H}, \mathrm{d}, J=1 \mathrm{~Hz})\end{array}$ \\
\hline 25 & 99 & $174-176$ & $\mathrm{C}_{17} \mathrm{H}_{23} \mathrm{~N}_{7} \mathrm{O}_{3}$ & $\begin{array}{r}54.68 \\
(54.63\end{array}$ & $\begin{array}{l}6.21 \\
6.29\end{array}$ & $\begin{array}{l}26.26 \\
26.08)\end{array}$ & 373 & $\begin{array}{l}1.74(4 \mathrm{H}, \mathrm{t}, J=6 \mathrm{~Hz}), 3.75(4 \mathrm{H}, \mathrm{t}, J=4 \mathrm{~Hz}), 3.84(4 \mathrm{H}, \mathrm{br} \mathrm{s}) \\
3.94(4 \mathrm{H}, \mathrm{brs}), 4.01(4 \mathrm{H}, \mathrm{s}), 7.08(\mathrm{lH}, \mathrm{s}), 7.76(1 \mathrm{H}, \mathrm{s}), 8.50 \\
(1 \mathrm{H}, \mathrm{s})\end{array}$ \\
\hline 26 & 64 & $160-161$ & $\mathrm{C}_{22} \mathrm{H}_{24} \mathrm{~N}_{8} \mathrm{O}$ & $\begin{array}{r}63.44 \\
(63.29\end{array}$ & $\begin{array}{l}5.81 \\
5.87\end{array}$ & $\begin{array}{l}26.90 \\
26.75)\end{array}$ & 416 & $\begin{array}{l}2.02(2 \mathrm{H}, \mathrm{dt}, J=4,13 \mathrm{~Hz}), 2.2-2.3(2 \mathrm{H}, \mathrm{m}), 3.3-3.4(2 \mathrm{H}, \mathrm{m}) \\
3.76(4 \mathrm{H}, \mathrm{t}, J=4 \mathrm{~Hz}), 3.86(4 \mathrm{H}, \mathrm{br}), 4.9-5.1(2 \mathrm{H}, \mathrm{m}), 7.10 \\
(1 \mathrm{H}, \mathrm{t}, J=1 \mathrm{~Hz}), 7.32-7.52(5 \mathrm{H}, \mathrm{m}), 7.77(1 \mathrm{H}, \mathrm{t}, J=1 \mathrm{~Hz}) \\
8.51(1 \mathrm{H}, \mathrm{t}, J=1 \mathrm{~Hz})\end{array}$ \\
\hline 27 & 53 & $119-120$ & $\mathrm{C}_{17} \mathrm{H}_{19} \mathrm{~N}_{7} \mathrm{O}$ & $\begin{array}{r}60.52 \\
(60.62\end{array}$ & $\begin{array}{l}5.68 \\
5.70\end{array}$ & $\begin{array}{l}29.06 \\
29.02)\end{array}$ & 337 & $\begin{array}{l}3.54(3 \mathrm{H}, \mathrm{s}), 3.7-3.8(8 \mathrm{H}, \mathrm{m}), 7.04(1 \mathrm{H}, \mathrm{s}), 7.2-7.4(5 \mathrm{H}, \mathrm{m}) \\
7.65(1 \mathrm{H}, \mathrm{brs}), 8.39(1 \mathrm{H}, \mathrm{brs})\end{array}$ \\
\hline 28 & 83 & $239-241$ & $\mathrm{C}_{14} \mathrm{H}_{19} \mathrm{~N}_{7} \mathrm{~S}_{2}$ & $\begin{array}{r}48.12 \\
(48.18\end{array}$ & $\begin{array}{l}5.48 \\
5.50\end{array}$ & $\begin{array}{l}28.06 \\
28.00)\end{array}$ & 349 & $\begin{array}{l}2.66(8 \mathrm{H}, \mathrm{t}, J=5 \mathrm{~Hz}), 4.14(8 \mathrm{H}, \mathrm{br} \mathrm{s}), 7.09(1 \mathrm{H}, \mathrm{d}, J=1 \mathrm{~Hz}) \\
7.75(1 \mathrm{H}, \mathrm{d}, J=1 \mathrm{~Hz}), 8.49(1 \mathrm{H}, \mathrm{s})\end{array}$ \\
\hline
\end{tabular}


br s), 3.7-3.9 (12H, m), 7.3-7.4 (5H, m). MS $m / z: 374\left(\mathrm{M}^{+}\right)$.

2-Chloro-4-(1,4-dioxa-8-azaspiro[4.5]decan-8-yl)-6-morpholino1,3,5-triazine (4h): $89 \%$ yield. Colorless crystals, mp 169-171 ${ }^{\circ} \mathrm{C}$. ${ }^{1} \mathrm{H}-\mathrm{NMR}\left(\mathrm{CDCl}_{3}\right) \delta: 1.72(4 \mathrm{H}, \mathrm{t}, J=6 \mathrm{~Hz}), 3.7-4.0(12 \mathrm{H}, \mathrm{m}), 3.99(4 \mathrm{H}$, s). MS $m / z: 341\left(\mathrm{M}^{+}\right)$.

2-Chloro-4-(4-cyano-4-phenylpiperidino)-6-morpholino-1,3,5-triazine (4i): $99 \%$ yield. Colorless crystals, $\mathrm{mp} 203-205^{\circ} \mathrm{C} .{ }^{1} \mathrm{H}-\mathrm{NMR}\left(\mathrm{CDCl}_{3}\right)$ $\delta: 1.99(2 \mathrm{H}, \mathrm{d}, J=13 \mathrm{~Hz}), 2.19(2 \mathrm{H}, \mathrm{d}, J=13 \mathrm{~Hz}), 3.32(2 \mathrm{H}, \mathrm{t}, J=13 \mathrm{~Hz})$, 3.6-3.9 (8H, m), 4.8-5.1 (2H, m) 7.3-7.5 (5H, m). MS $m / z: 384\left(\mathrm{M}^{+}\right)$.

2-Chloro-4-( $N$-methyl- $N$-phenylamino)-6-morpholino-1,3,5-triazine (4j): $84 \%$ yield. Colorless crystals, mp 97-100 ${ }^{\circ} \mathrm{C} .{ }^{1} \mathrm{H}-\mathrm{NMR}\left(\mathrm{CDCl}_{3}\right)$ $\delta: 3.49(3 \mathrm{H}, \mathrm{s}), 3.6-3.9(8 \mathrm{H}, \mathrm{m}), 7.2-7.5(5 \mathrm{H}, \mathrm{m}) . \mathrm{MS} m / z: 305\left(\mathrm{M}^{+}\right)$.

These were used for the next reaction without further purification.

General Procedure for Preparation of Synthesis of Triamino-substituted 1,3,5-Triazines I, II, III and IV Synthesis of 2-(1-Imidazolyl)-4,6dimorpholino-1,3,5-triazine (17): Imidazole $(0.694 \mathrm{~g}, 10.2 \mathrm{mmol})$ and $\mathrm{NaOH}(0.41 \mathrm{~g}, 10.3 \mathrm{mmol})$ was added to a solution of $1 \mathrm{~d}(1.44 \mathrm{~g}$, $5.04 \mathrm{mmol})$ in DMF $(10 \mathrm{ml})$ at room temperature. The reaction mixture was stirred at $110^{\circ} \mathrm{C}$ for $30 \mathrm{~min}$, then extracted with AcOEt. The extract was washed with brine, dried over $\mathrm{MgSO}_{4}$ and evaporated in vacuo. The residue was purified by silica gel column chromatography with $\mathrm{CH}_{2} \mathrm{Cl}_{2}-\mathrm{MeOH}(95: 5)$ to afford $\mathbf{1 7}(1.36 \mathrm{~g}, 85 \%)$ as colorless crystals.

Compounds 5-16, 18 and 20-28 were obtained by a similar procedure to that described for 17.

2-Dimethylamino-4,6-dimorpholino-1,3,5-triazine (29) Morpholine $(0.18 \mathrm{~g}, 2.1 \mathrm{mmol})$ and $\mathrm{NaOH}(0.084 \mathrm{~g}, 2.1 \mathrm{mmol})$ were added to a solution of $3(0.45 \mathrm{~g}, 2.0 \mathrm{mmol})$ in DMF $(10 \mathrm{ml})$ at room temperature. The reaction mixture was stirred at $110^{\circ} \mathrm{C}$ for $30 \mathrm{~min}$, then extracted with AcOEt. The extract was washed with brine, dried over $\mathrm{MgSO}_{4}$ and evaporated in vacuo. The residue was purified by silica gel column chromatography with $\mathrm{CH}_{2} \mathrm{Cl}_{2}-\mathrm{MeOH}(95: 5)$ to afford $29(0.18 \mathrm{~g}, 31 \%)$ as colorless crystals, mp $188-190{ }^{\circ} \mathrm{C} .{ }^{1} \mathrm{H}-\mathrm{NMR}\left(\mathrm{CDCl}_{3}\right) \delta: 3.09(6 \mathrm{H}, \mathrm{s}), 3.7-3.9$ $(16 \mathrm{H}, \mathrm{m})$. MS $m / z: 294\left(\mathrm{M}^{+}\right)$.

The properties of the prepared compounds 5-18 and 20-28 are shown in Table 2.

Aromatase-Inhibitory Activity The inhibitory activity was measured by the method of Thompson and Siiteri. ${ }^{2 a)}$ Briefly, the assay measures the loss of tritium to the aqueous phase of the incubation medium during aromatization of androstenedione ${ }^{3} \mathrm{H}$-labeled at positions $\mathrm{l}$ and 2 . Test compounds were dissolved in dimethyl sulfoxide (DMSO) and further dilution was done with $3 \%$ DMSO $(\mathrm{v} / \mathrm{v})$ in $0.05 \mathrm{M}$ phosphate buffer $(\mathrm{pH}$ 7.4). The reaction mixture contained $\left[1 \beta, 2 \beta-{ }^{3} \mathrm{H}\right]-4$-androstene-3,17dione $(500 \mathrm{pmol})$, human placental microsomes $(20 \mu \mathrm{g}$ protein $)$, and various concentrations of test compound $\left(10^{-4}-10^{-9}\right)$ in phosphate buffer in a final volume of $450 \mu$ l. The preincubation was carried out at $37^{\circ} \mathrm{C}$ for $3 \mathrm{~min}$. The reaction was started by the addition of NADPH $(125 \mathrm{nmol} / 5 \mu \mathrm{l})$ and stopped after $15 \mathrm{~min}$ of incubation by the addition of $25 \%$ trichloroacetic acid $(50 \mu \mathrm{l})$. A suspension of activated charcoal ( $50 \mu \mathrm{l}$, containing $5 \%$ activated charcoal and $0.5 \%$ dextolane) was added to the reaction mixture. The resulting mixture was vortexed for $30 \mathrm{~min}$ and centrifuged at $600 \times \boldsymbol{g}$ for $5 \mathrm{~min}$. After centrifugation, $400 \mu \mathrm{l}$ of aqueous phase was dissolved in Aquazol-2 $(5 \mathrm{ml})$ and the radioactivity of the resulting solution was measured with a liquid scintillation spectrometer (Aloka LSC-3100). Thus, the aromatase-inhibitory activities of the test compounds were radiometrically determined. The $\mathrm{IC}_{50}$ was determined by using an inhibition curve constructed by plotting the reciprocal percent conversion versus the concentration of the test compound.

$\mathbf{P 4 5 0}_{\mathrm{Scc}}$-Inhibitory Activity The inhibitory activity was measured by the method of Shikita and Hall. ${ }^{17)}$ Briefly, the assay radiometrically measures the rate of transformation to the organic phase of the incubation medium during the biosynthetic transformation of cholesterol to pregnenolone. Test compounds were dissolved in DMSO and further dilution was done with $2 \%$ DMSO $(v / v)$ in $0.05 \mathrm{~m}$ phosphate buffer $(\mathrm{pH}$ 7.4). The reaction mixture contained $\left[4-{ }^{14} \mathrm{C}\right]$ cholesterol $(5 \mathrm{nmol} / 250$ $\mathrm{Bq} / 10 \mu \mathrm{l}$ ethanol), sonicated pig adrenal mitochondria (320 $\mu \mathrm{g}$ protein) and various concentrations $\left(10^{-4}-10^{-7} \mathrm{M}\right)$ of test compound in phosphate buffer in a final volume of $900 \mu \mathrm{l}$. The preincubation was carried out at $37^{\circ} \mathrm{C}$ for $3 \mathrm{~min}$. The reaction was started by the addition of NADPH $(250 \mathrm{nmol} / 100 \mu \mathrm{l})$ and stopped after $15 \mathrm{~min}$ by the addition of $\mathrm{CH}_{2} \mathrm{Cl}_{2}(3 \mathrm{ml})$. Following extraction and centrifugation, the organic layer was removed and evaporated to dryness. The residue was taken up in $\mathrm{CH}_{2} \mathrm{Cl}_{2}$, and chromatographed on a silica gel (13181 Silicagel Kodak) thin layer plate with AcOEt-hexane (3:7, v/v). The radioactive spots were identified by comparison with authentic standards and quantified by the use of a liquid scintillation spectrometer (Aloka

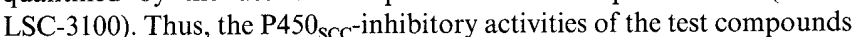
were radiometrically determined. $\mathrm{The} \mathrm{IC}_{50}$ was determined by using an inhibition curve constructed by plotting the reciprocal percent conversion versus the concentration of the test compound.

\section{References}

1) a) Brodie A. M. H., Biochem. Pharmacol., 34, 3213-3219 (1985); b) Van Wauwe J. P., Janssen P. A. J., J. Med. Chem., 32, 2231-2239 (1989); c) Cole P. A., Robinson C. H., ibid., 33, 2933-2942 (1990).

2) a) Thompson E. A., Jr., Siiteri P. K., J. Biol. Chem., 249, 53645372 (1974); b) Henderson D., Norbisrath D., Kerb U., J. Steroid Biochem., 24, 303-306 (1986).

3) Griffiths C. T., Hall T. C., Saba Z., Barlow J. J., Nevinny H. B., Cancer, 32, 31-37 (1973).

4) Fishman L. M., Liddle G. W., Island D. P., J. Clin. Endocrinol. Metabolism, 27, 481-490 (1967).

5) a) Foster A. B., Jarman M., Leung C-S., Rowlands M. G., Taylor G. N., J. Med. Chem., 26, 50-54 (1983); b) Shaw M. A., Nicholls P. J., Smith H. J., J. Steroid Biochem., 31, 137-146 (1988).

6) Uzgiris V. I., Whipple C. A., Salhanick H. A., Endocrinology, 101, 89-92 (1977).

7) Steele R. E., Mellor L. B., Sawyer W. K., Wasvary J. M., Browne L. J., Steroids, 50, 147-161 (1987).

8) Goldin A., Wolpert-Defilippes M. K., Bull. Cancer, 66, 61-66 (1979).

9) Foster B. J., Harding B. J., Leyland-Jones B., Hoth D., Cancer Treatment Rev., 13, 197-217 (1986).

10) Jones C. D., Winter M. A., Hirsch K. S., Stamm N., Taylor H. M., Holden H. E., Davenport J. D., Krumkalns E. V., Suhr R. G., J. Med. Chem., 33, 416-429 (1990).

11) Quirke J. M. E., "Comprehensive Heterocyclic Chemistry," Vol. 3, ed. by Katritzky A. R., Rees C. W., Pergamon Press, Oxford, 1984, pp. 457-530.

12) Thurston J. T., Dudley J. R., Kaiser D. W., Hechenbleikner I., Schaefer F. C., Holm-Hansen D., J. Am. Chem. Soc., 73, 2981$2983(1951)$.

13) Wamhoff H., "Comprehensive Heterocyclic Chemistry," Vol. 5, ed. by Katritzky A. R., Rees C. W., Pergamon Press, Oxford, 1984, pp. $678-680$.

14) Browne L. J., Gude C., Rodriguez H., Steele R. E., Bhatnagar A. S., J. Med. Chem., 34, 725-736 (1991).

15) Kaiser D. W., Thurston J. T., Dudley J. R., Schaefer F. C., Hechenbleikner I., Holm-Hansen D., J. Am. Chem. Soc., 73, 2984-2986 (1951).

16) Detweiler W. K., Amstutz E. D., J. Am. Chem. Soc., 74, 14831485 (1952).

17) Shikita M., Hall P. F., Proc. Nat. Acad. Sci. U.S.A., 71, 14411445 (1974). 